\title{
Optical microbubble resonator
}

\author{
M. Sumetsky, ${ }^{*}$ Y. Dulashko, and R. S. Windeler \\ OFS Laboratories, 19 Schoolhouse Road, Somerset, New Jersey 08873, USA \\ *Corresponding author: sumetski@ofsoptics.com
}

Received December 7, 2009; accepted January 29, 2010;

posted February 18, 2010 (Doc. ID 121139); published March 17, 2010

\begin{abstract}
We develop a method for fabricating very small silica microbubbles having a micrometer-order wall thickness and demonstrate the first optical microbubble resonator. Our method is based on blowing a microbubble using stable radiative $\mathrm{CO}_{2}$ laser heating rather than unstable convective heating in a flame or furnace. Microbubbles are created along a microcapillary and are naturally opened to the input and output microfluidic or gas channels. The demonstrated microbubble resonator has $370 \mu \mathrm{m}$ diameter, $2 \mu \mathrm{m}$ wall thickness, and a $Q$ factor exceeding $10^{6}$. (C) 2010 Optical Society of America

OCIS codes: 230.3990, 220.4000, 060.2340, 140.4780, 140.3948, 130.6010.
\end{abstract}

Investigation and application of the high $Q$-factor optical microresonators has become an interesting and rapidly developing field of modern optics. Special attention is given to the Fabry-Perot, ring, disk, sphere, and toroid microresonators $[1,2]$ as well as more recently introduced bottle [3-5], coil [6,7], and capillary $[8,9]$ microresonators. Optical microresonators are demonstrated as super-perceptive physical, chemical, and biological sensors and microlasers and have promising applications in cavity quantum electrodynamics, cavity optomechanics, and other areas of fundamental and applied research and development $[1,2]$.

In this Letter we experimentally demonstrate a type of optical microresonator-a microbubble resonator. This resonator is created from a silica microbubble having a diameter of a few hundred micrometers and a micrometer-order wall thickness. The blowing of silica bubbles that are so small and thin is challenging. In fact, consider an open silica tube filled with pressurized gas. For a constant pressure, the process of blowing of a thin-wall bubble from a section of this tube, heated in a flame or furnace, is unstable and, eventually, will result in breakage. For a very small tube diameter and bubble dimensions, the accurate control of pressure becomes extremely difficult. Here, we suggest a method that stabilizes the expansion of a bubble.

Our approach is based on application of the stable radiative heating by a $\mathrm{CO}_{2}$ laser beam rather than the unstable convective heating in a flame or furnace. This approach originates from the idea developed in microfiber tapering using a $\mathrm{CO}_{2}$ laser $[10,11]$. It has been found that a fiber heated with a laser beam of certain power can be tapered down to a certain threshold diameter, $d_{t h}$, only. This effect is never observed for conventional fiber tapering in a flame and can be used for controlling the tapered fiber diameter with the laser beam power [11]. Freezing a microfiber at the diameter $d_{t h}$ has a simple explanation $[10,11]$. The $\mathrm{CO}_{2}$ laser beam penetration depth into the heated silica is a few tens of micrometers [12]. Therefore, for a thin silica fiber having a diameter $d$ $\leqslant 10 \mu \mathrm{m}$, the beam power is acquired by the silica volume almost uniformly [Fig. 1(a)]. For this reason, in the process of fiber tapering, the acquired power decreases proportionally to the heated volume or to the $d^{2}$. On the other hand, the dissipated power is always proportional to the fiber surface area that decreases proportionally to the fiber diameter $d$, i.e., slower than the acquired power. Thus, the steadystate temperature of the fiber heated with the $\mathrm{CO}_{2}$ laser decreases with its diameter.

The blowing of a silica microbubble can be controlled similarly. The thickness of the bubble wall, $w$, decreases in process of the bubble expansion. For a given incident laser beam power, the wall thickness cannot be less than the threshold value, $w_{t h}$, for the following reason: the wall with thickness $w \leqslant 10 \mu \mathrm{m}$ is heated almost uniformly [12]. Ignoring, for simplicity, the gas dynamics inside the microcapillary, we conclude that the acquired power is proportional to $w$, while the dissipated power is independent of $w$ [Fig. 1(b)]. As the result, the total heating effect decreases with $w$ and the microbubble freezes at a threshold wall thickness $w_{t h}$.

Generally, if the characteristic dimensions of an object are less than the depth of radiation penetration, then the acquired power decreases proportionally to the volume of this object. The dissipated power of the object is always proportional to its surface area. For this reason, the heating rate decreases with volume faster than the cooling rate so that the steady temperature of a small object heated by radiation decreases with its dimensions. Alternatively, the power acquired through the convective heating, similar to
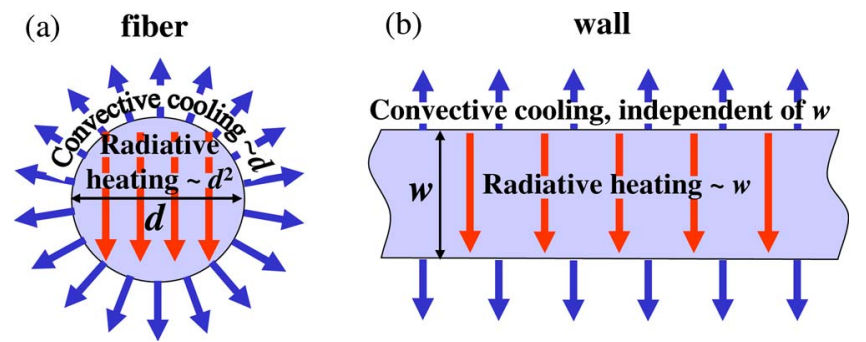

Fig. 1. (Color online) Illustration of radiative heating accompanied by convective cooling (a) for a thin fiber and (b) for a thin wall. 
the dissipated power, is proportional to the surface area and the cooling effect is not observed.

A microbubble is blown from a microcapillary that is fabricated from a silica tube preform at a conventional fiber-drawing station. The microcapillary is pressurized and rotated inside the incident beam of a $\mathrm{CO}_{2}$ laser. The optical microscope image of the fabricated sample silica microbubble is shown in Fig. 2(a). This bubble is created from a capillary with diameter $D_{\text {cap }}=120 \mu \mathrm{m}$ and wall thickness $w_{\text {cap }}=6.3 \mu \mathrm{m}$. The microbubble wall thickness $w_{b}$ is estimated from its average diameter $D_{b} \approx 370 \mu \mathrm{m}$ by the relation $w_{b}$ $=w_{c a p} D_{c a p} / D_{b}$, yielding $w_{b} \approx 2 \mu \mathrm{m}$. The developed method allows sequential blowing of microbubbles with similar and different diameters and wall thickness. This can be achieved by changing the power and uniformity of the input beam as well as the diameter and wall thickness of the capillary. As an example, Fig. 2(b) shows an image of three microbubbles spaced by $500 \mu \mathrm{m}$ having a wall thickness of $\sim 1.5 \mu \mathrm{m}$. By increasing the laser beam power we are able to fabricate larger microbubbles having a submicrometer wall thickness.

Following experiments with microsphere resonators $[1,2,13]$, we investigate the resonant properties of a microbubble by coupling it to a microfiber. A biconical taper with a microfiber waist is fabricated from a conventional single-mode fiber by adiabatic tapering using the $\mathrm{CO}_{2}$ laser indirect heating method [14]. The input and output ends of the taper are connected to the JDS Uniphase tunable laser source and detector, respectively. The diameter of the microfiber section coupled to the microbubble is $\sim 2 \mu \mathrm{m}$. As an example, the black curve 1 in Fig. 3 plots the transmission spectrum of the microbubble shown in Fig. 2(a) (microfiber position A) measured in the wave-
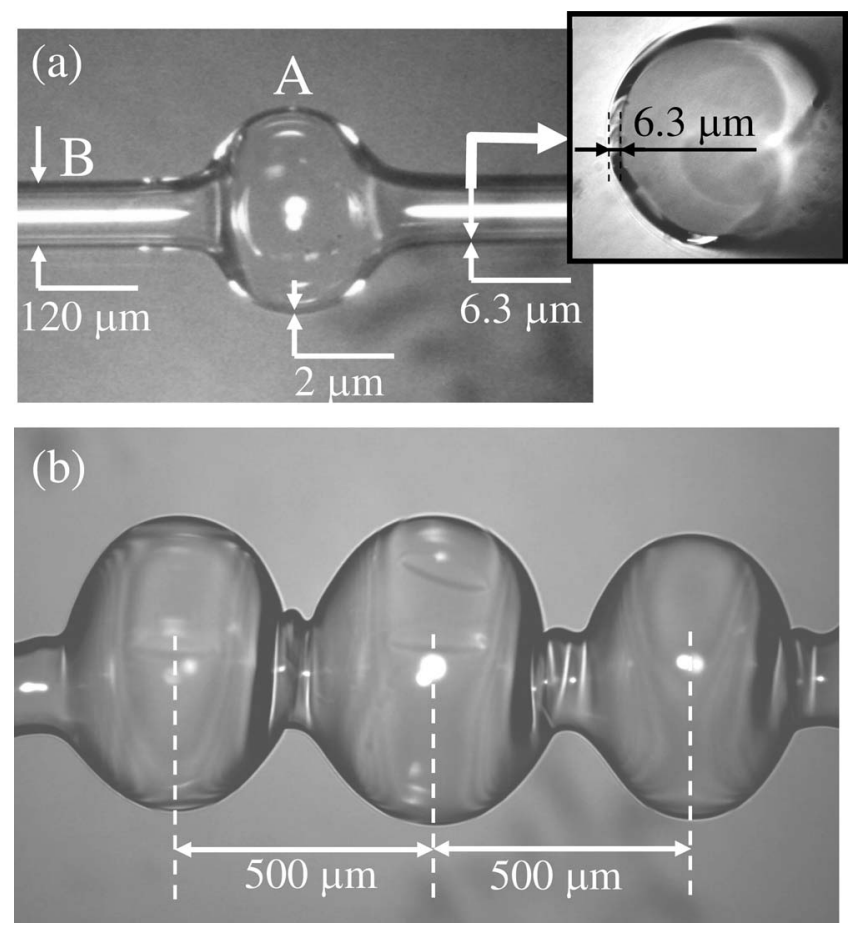

Fig. 2. (a) Optical microscope image of a microbubble; (b) optical microscope image of three successive microbubbles.

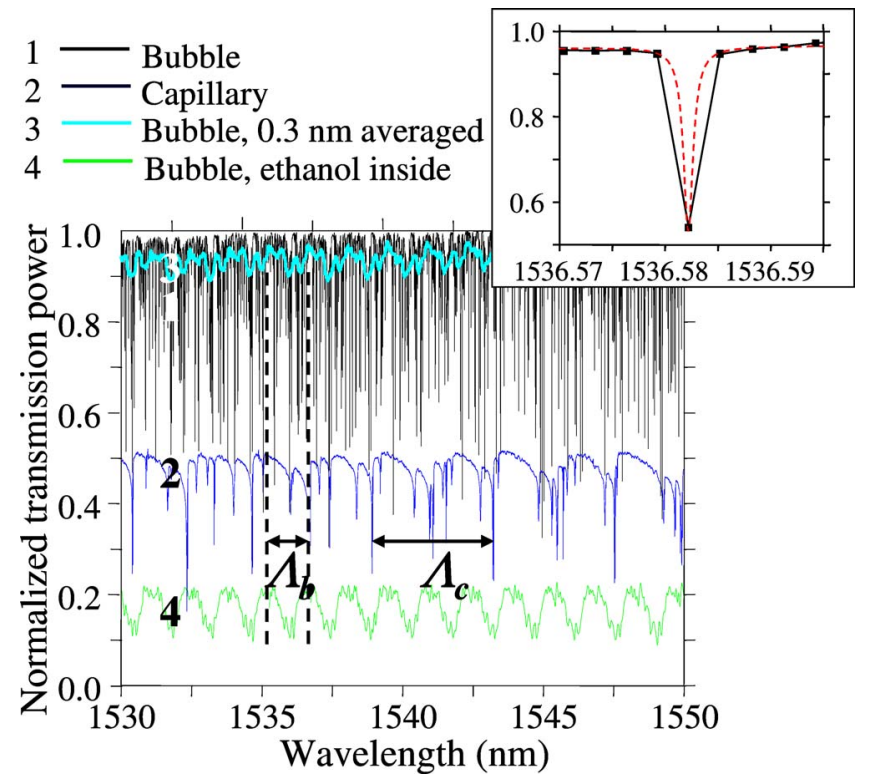

Fig. 3. (Color online) Transmission spectra of the microbubble and microcapillary shown in Fig. 2(a): 1, microbubble; 2 , capillary; 3 , spectrum of the microbubble averaged over $0.3 \mathrm{~nm}$ interval; 4, microbubble filled with ethanol. Inset, magnified measurement data near a resonant peak (dots) fitted with a Lorentzian curve (dashed).

length interval from 1530 to $1550 \mathrm{~nm}$. For comparison, the blue curve 2 shows the transmission spectrum of the capillary (microfiber position B). The inset in Fig. 3 magnifies the microbubble spectrum near a resonance. This inset determines the lowest estimate of the resonance $Q$ factor, $Q=1.5 \times 10^{6}$, which is obtained by Lorentzian fit of the experimental data limited by the $3 \mathrm{pm}$ resolution of our measurement system. The cyan curve 3 in Fig. 3 characterizes the density of the microbubble transmission spectrum. It is obtained by averaging of the black curve 1 over 100 measurement points $(0.3 \mathrm{~nm}$ interval). While the distribution of peaks of the black curve 1 looks stochastic, the cyan curve 3 has a period $\Lambda_{b}$ determined by the microbubble circumference length. The ratio of this period and the period $\Lambda_{c}$ of the capillary spectrum (blue curve 2) determines the ratio of the microcapillary and the microbubble diameters $\Lambda_{c} / \Lambda_{b} \approx 0.32$, in good agreement with the measurement taken from Fig. 2(b). Finally, the green curve 4 in Fig. 3 determines the oscillations of the microbubble transmission spectrum after filling it with ethanol (refractive index 1.36). As expected, the period of these oscillations coincide with the period of the spectrum density oscillations of the empty microbubble. In this case, the disappearance of high- $Q$ resonances is caused by the ethanol transmission loss.

Compared with other microresonators, there are important advantages of a microbubble resonator in the application for microfluidic sensing. In fact, as opposed to microsphere or microtoroid resonators and similar to a microcapillary resonator $[8,9]$, the tested liquid or gas flows inside the microbubble and does not disturb the microfiber alignment. Furthermore, as opposed to the microcapillary, the mi- 
crobubble possesses real localized eigenmodes and does not exhibit noticeable insertion loss (compare black curve 1 and blue curve 2). Several other applications of a microbubble resonator can be considered. For example, it will be interesting to investigate the tunability of a microbubble resonator under mechanical deformations $[15,5]$ as well as its resonant optomechanical behavior [16].

In summary, a novel type of optical microresonator, a microbubble resonator, is created. The developed method of silica microbubble fabrication is based on radiative heating with the $\mathrm{CO}_{2}$ laser. It allowed us to fabricate microbubbles that are naturally opened to the input and output microcapillary channels. The demonstrated microbubble resonator has $370 \mu \mathrm{m}$ diameter, $2 \mu \mathrm{m}$ wall thickness, and a $Q$ factor exceeding $10^{6}$. Potentially, it is possible to create microbubbles of different shapes and wall thicknesses by variation of the power and uniformity of the laser beam, asymmetry and speed of rotation, and parameters of the capillary. We expect applications of the microbubble resonator as a microfluidic bio and chemical sensor as well as a tunable laser, qualitatively improving the performance of microcapillarybased microfluidic devices demonstrated so far. Potential applications of the demonstrated silica microbubble can go far beyond utilizing its high $Q$-factor resonance behavior.

The authors are grateful to D. J. DiGiovanni for numerous discussions and suggestions.

\section{References}

1. K. J. Vahala, Nature 424, 839 (2003).

2. A. B. Matsko, ed., Practical Applications of Microresonators in Optics and Photonics (CRC Press, 2009).

3. M. Sumetsky, Opt. Lett. 29, 8 (2004).

4. G. S. Murugan, J. S. Wilkinson, and M. N. Zervas, Opt. Express 17, 11916 (2009).

5. M. Pöllinger, D. O'Shea, F. Warken, and A. Rauschenbeutel, Phys. Rev. Lett. 103, 053901 (2009).

6. M. Sumetsky, Opt. Express 12, 2303 (2004).

7. M. Sumetsky, J. Lightwave Technol. 26, 21 (2008).

8. I. M. White, H. Oveys, and X. Fan, Opt. Lett. 31, 1319 (2006).

9. I. M. White, S. I. Shopova, H. Zhu, J. D. Suter, Y. Sun, and X. Fan, in Advanced Photonic Structures for Biological and Chemical Detection, X. Fan, ed. (Springer, 2009), pp. 377-393.

10. A. J. C. Grellier, N. K. Zayer, and C. N. Pannell, Opt. Commun. 152, 324 (1998).

11. T. E. Dimmick, G. Kakarantzas, T. A. Birks, and P. St. J. Russell, Appl. Opt. 38, 6845 (1999).

12. A. D. McLachlan and F. P. Meyer, Appl. Opt. 26, 1728 (1987).

13. J. C. Knight, G. Cheung, F. Jacques, and T. A. Birks, Opt. Lett. 22, 1129 (1997).

14. M. Sumetsky, Y. Dulashko, and A. Hale, Opt. Express 12, 3521 (2004).

15. W. von Klitzing, R. Long, V. S. Ilchenko, J. Hare, and V. Lefévre-Seguin, Opt. Lett. 26, 166 (2001).

16. T. J. Kippenberg and K. J. Vahala, Opt. Express 15, 17172 (2007). 